\title{
Auditory continuity effects with binaural stimuli
}

\author{
WILLARD R. THURLOW \\ University of Wisconsin, Madison, Wisconsin
}

\begin{abstract}
The term "continuity effect" has been used to refer to a situation in which one auditory stimulus, $A$, alternating in time with another stimulus, $B$, is perceived as continuous, even though $A$ is not physically continuous. This effect has previously been studied in cases in which $B$ was higher in intensity than $A$; it has been hypothesized that $B$ causes continuity in $A$ when it removes the $A$ gap-cues. In the present experiments, continuity effects were studied with binaural noise, tone, and speech stimuli. It was shown that intensity relations of $B$ and $A$ stimuli in each ear, rather than total binaural input of $B$ as compared with total binaural input of $A$, are utilized in determining the final continuity effect. Results can be described by the general hypothesis that when no gap can be detected in a stimulus, in either ear, there is a synthesis of available information about that stimulus over a longer time period than when gaps are detected. Finally, two additional, special, types of binaural continuity effects are described and illustrated.
\end{abstract}

The term " continuity effect" " has been used to describe a situation in which a stimulus, $A$, alternated in time with a more intense stimulus, $B$, is heard as continuous (even though it is not physically continuous). The extensive literature on this effect has been summarized previously (see, e.g., Verschurre, 1978; Warren, 1982).

In most of these studies, the stimuli have been to one ear. Some studies, however, have presented $A$ and $B$ alternating between the ears ( $A$ in one ear, $B$ in the other) and found continuity (Elfner \& Caskey, 1965; Elfner \& Homick, 1966, 1967). These continuity effects are weak relative to monaural effects. That is, only $A$ stimuli that have gaps of short duration can be made to sound continuous by the sounding of a $B$ stimulus in the other ear during the gaps in $A$. It has been suggested that this gapfilling may be related to a cross-ear facilitation effect from $B$, which causes continued neural firing of $A$ during the gap in $A$ (Elfner \& Caskey, 1965; Thurlow \& Elfner, 1959a).

The present experiments studied situations in which continuity effects for binaural noise, tone, and speech stimuli can be obtained over much longer durations.

\section{Preliminary Observations}

With reference to the schematic diagram in Figure 1 , $B$ and $\boldsymbol{A}$ represent binaural noise stimuli, alternating in time; $\mathrm{L}$ and $\mathrm{R}$ represent left and right, respectively. $A \mathrm{~L}$ was set at a level $10 \mathrm{~dB}$ lower than $B \mathrm{~L}, B \mathrm{R}$, and $A \mathrm{R}$. The latter stimuli were at a level of $64 \mathrm{~dB}(\mathrm{~A})$. (DB(A) represents decibels obtained with the " $A$ " filter setting of the sound-level meter.) Under these initial conditions, the $B$ stimulus is perceived in the median plane ("center') sounding intermittently. The $A$ stimulus is perceived to the right and as continuous (even though it is not physically continuous).

Requests for reprints should be sent to Willard Thurlow, Department of Psychology, University of Wisconsin, 1202 West Johnson St., Madison, WI 53706.
When $B R$ is decreased in intensity slightly (by 1-2 dB), the $A$ sound to the right becomes intermittent. When $B R$ is increased in intensity, the $A$ sound can be perceived as remaining continuous. The fact that a small decrease in $B R$ causes the sound heard to the right to appear intermittent indicates that binaural continuity perception is being controlled by intensity relations in each ear separately-because a small decrease in $B R$ would still leave total neural input of $B$ larger than total neural input of $A$. If continuity were controlled by relations between total $B$ and $A$ inputs, we would not expect these results.

In Experiment 1, these same stimuli were used to obtain measurements with samples of undergraduate subjects.

\section{EXPERIMENT 1}

\section{Method}

Apparatus and Stimuli. $A$ and $B$ stimuli to right and left ears are diagramed schematically in Figure 1 . The dashed line for $A \mathrm{~L}$ indicates schematically that the $A \mathrm{~L}$ stimulus was set at a level $10 \mathrm{~dB}$ lower than the other stimuli.

Output of a noise generator (Grason-Stadler 455C) was led through a mixer to two electronic switches (Grason-Stadler 829D). Timing of the switches was controlled by a Psionix 2400 system, which alternated presentations of $A$ and $B$ stimuli. $B$ stimuli were then

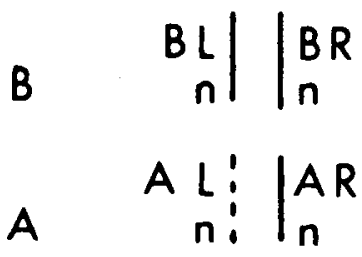

Figure 1. Schematic diagrams of initial stimulus conditions. B and A represent stimuli alternating in time, delivered to left $(L)$ and right (R) earphones. The right-hand line of a pair represents a stimulus delivered to the right earphone; the left-hand line of a pair represents a stimulus delivered to the left earphone. Other symbols: $n=$ noise; a dashed line represents a lower intensity than a solid line. 
led through attenuators and mixers to left and right ears ( $B \mathrm{~L}$ and $B \mathrm{R}$ ), as were $A$ stimuli ( $A \mathrm{~L}$ and $A \mathrm{R}$ ). Earphones were Telephonics, Model TDH-39.

In Experiment 1a, time for onset and offset of stimuli was set at $1 \mathrm{msec}$. In Experiment $1 \mathrm{~b}$, it was set at $5 \mathrm{msec}$. Triggering of the electronic switches, however, was arranged so that $A$ and $B$ stimuli did not overlap in time. Durations of $A$ and $B$ stimuli were $41 \mathrm{msec}$ in Experiment $1 \mathrm{a}$ and $45 \mathrm{msec}$ in Experiment $\mathrm{lb}$. The level of noise (except for $A \mathrm{~L}$, which was attenuated $10 \mathrm{~dB}$ ) was at $64 \mathrm{~dB}$, as measured with a Model 1560-P82 General Radio coupler and B \& K Model 2203 sound-level meter, using the " $A$ " filter setting.

Subjects and Procedure. Subjects in both experiments (as in all of our experiments) were undergraduate volunteers ( 9 in Experiment $1 \mathrm{a}, 10$ in Experiment $1 b$ ).

First, a demonstration was given to each subject of what was called "continuous" noise and what was called "interrupted" noise by having the subject listen to the left-earphone stimuli alone when $B \mathrm{~L}$ and $A \mathrm{~L}$ were of equal intensity ("continuous") and then when 10-dB attenuation was added to AL ("interrupted"). The subject was instructed to listen for the particular rate characteristic of the interrupted stimuli.

For the main part of the experiment, changes were introduced in $A R$ and $B R$ stimuli. When $B R$ was decreased in intensity and when $A \mathrm{R}$ was increased in intensity, the subject was asked to signal as soon as he/she heard the noise to the right become intermittent at the rate he/she had been asked to listen for. When $B \mathrm{R}$ was increased in intensity and $A \mathrm{R}$ was decreased in intensity, the subject was asked to signal as soon as the continuous noise on the right could no longer be heard. (The latter intensity changes cause the perceived location of the $A$ and $B$ stimuli to come together, with the resulting domination of perception by the intermittent $B$ stimulus.) In Experiment 1b, two measures were obtained for each subject for each condition, using the method of limits; in Experiment la, one measure was obtained for each subject for each condition.

\section{Results}

Results for Experiments $1 \mathrm{a}$ and $1 \mathrm{~b}$ are shown in Table 1 . It is clear that the average increase in $A \mathrm{R}$ and average decrease in $B R$, to produce perception of interruption, are both small. On the other hand, the increase in $B R$ and decrease in $A R$, necessary before perception of the continuous stimulus to the right was obscured, are both larger. Signed ranks tests showed an asymmetry: $B \mathrm{R}$ increase $>B \mathrm{R}$ decrease and $A \mathrm{R}$ decrease $>A \mathrm{R}$ increase $(p<.01)$. Erchul (1979) noted a similar asymmetry, but thought that both increases and decreases in $A \mathrm{R}$ (and $B \mathrm{R}$ ) produced intermittency; the results appeared puzzling. The most important conclusion from the present results, however, is not that there was an asymmetry, but that continuity in the $A$ stimulus was perceived unless $A \mathrm{R}$ exceeded $B R$ in intensity.

We checked with the subjects of Experiment $1 \mathrm{~b}$, and found that the subjective location of the continuous sound could be changed by adding a time lead in the right ear. The subjective location of the continuous sound to the right

Table 1

Average Decibel Changes to Produce Effects on A Sound of Figure 1

\begin{tabular}{|c|c|c|c|c|}
\hline \multirow[b]{2}{*}{ Experiment } & \multicolumn{2}{|c|}{ Interruption Perceived } & \multicolumn{2}{|c|}{ Continuous Sound Lost } \\
\hline & $A \mathrm{R}$ Increase & BR Decrease & $A \mathrm{R}$ Decrease & $B$ R Increase \\
\hline la & 1. & 2 & 6 & 6 \\
\hline $1 b$ & 1.4 & 2.0 & 5.0 & 6.9 \\
\hline
\end{tabular}

B<smiles>[In]I</smiles>

A

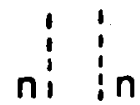

Figure 2. Schematic diagrams of initial stimulus conditions. B and A represent stimuli alternating in time, delivered to left and right earphones. The right-hand line of a pair represents a stimulus delivered to the right earphone; the left-hand line of a pair represents a stimulus delivered to the left earphone. Other symbols: $n=$ noise; a dashed line represents a lower intensity than a solid line.

is determined by the intensity and time difference cues that determine the location of the $A$ sound alone.

That interruption is perceived with a small decrease in $B R$ intensity would not have been predicted from a model that describes continuity effects in terms of relations between total magnitude of neural effects from $A$ and $B$ stimuli, because total $B$ neural effect should still be larger than total $A$ neural effect, even after a small decrease in $B R$ intensity. A further demonstration that the final continuity or intermittence perception is not based on simple addition of components from each ear can be obtained by using the noise pattern of Figure 1. After the subjects listened to this pattern and matched the loudness of $A$ and $B$ stimuli by increasing the intensity of $A \mathrm{R}$, the $B$ stimulus was moved over to the subjective location of the $A$ stimulus by the introduction of a time lead for $B R$. We were able to get the stimuli in the same subjective leftright location with 8 of the 12 subjects tested. These subjects heard, paradoxically, two stimuli in the same place, each intermittent.

\section{EXPERIMENT 2}

The stimulus situation used in this experiment is shown in Figure 2. Noise stimuli were used, as in Experiment 1, with $B$ and $A$ alternating in time. $B \mathrm{~L}, A \mathrm{R}$, and $A \mathrm{~L}$ were set initially at $10 \mathrm{~dB}$ less than $B R$. $B$ R was at $64 \mathrm{~dB}(\mathrm{~A})$. Because of the intensity relations at the two ears, the $B$ stimulus is heard toward the right and the $A$ stimulus is heard in the median plane ("center"). In this experiment, observations were made on the center noise. In the center noise, there is both a left-ear component, $A \mathrm{~L}$, and a rightear component, $A \mathrm{R}$. Initially the center noise should sound continuous, because $B \mathrm{~L}=A \mathrm{~L}$, and $B \mathrm{R}>A \mathrm{R}$. But if $B \mathrm{~L}$ is decreased in intensity (or $A \mathrm{~L}$ increased) so that $B \mathrm{~L}<$ $A \mathrm{~L}$, interruptions in the center noise should be detectable.

\section{Method}

The subjects were 10 undergraduate volunteers. Apparatus and stimulus timing were the same as in Experiment $1 \mathrm{~b}$.

To gain an understanding of what was meant by "continuous" and "interrupted" noise, each subject first listened to the left earphone alone when $B \mathrm{~L}$ and $A \mathrm{~L}$ were equal in intensity ("continuous") and when $5 \mathrm{~dB}$ was added to $B \mathrm{~L}$ ("interrupted"). The subject was asked to listen for this particular rate of interruption. 
Under the initial stimulus conditions of this experiment (diagramed in Figure 2), the subjects heard a continuous noise in the median plane. The intensity of $B \mathrm{~L}$ or $A \mathrm{~L}$ was then changed. When $B \mathrm{~L}$ was decreased (or $A \mathrm{~L}$ increased), the subject was asked to report as soon as he/she perceived the center noise to be interrupted. When $B \mathrm{~L}$ was increased (or $A \mathrm{~L}$ decreased), the subject was asked to report as soon as the continuous noise disappeared. (When $B \mathrm{~L}$ is increased in intensity, the subjective location of the $B$ stimulus approaches that of the $A$ stimulus, and the intermittent $B$ stimulus then dominates the continuous $A$ sound; when $A \mathrm{~L}$ is decreased in intensity, the subjective location of the $A$ stimulus approaches that of the $B$ stimulus, and again the intermittent $B$ stimulus dominates the continuous $A$ sound.) Two measures were obtained for each condition, using the method of limits.

\section{Results}

Table 2 shows average results. The average decrease in $B \mathrm{~L}$ and average increase in $A \mathrm{~L}$, to produce perception of interruption in the center noise, are both small. The average increase in $B \mathrm{~L}$ and average decrease in $A \mathrm{~L}$, before perception of the continuous $A$ sound was lost, were both larger. Signed ranks tests showed that the $B L$ decrease was significantly smaller than the $B \mathrm{~L}$ increase and that the $A \mathrm{~L}$ increase was significantly smaller than the $A \mathrm{~L}$ decrease. Of course, the asymmetry is only an aspect of the more important result that interruption in the center noise was perceived only when $B \mathrm{~L}<A \mathrm{~L}$.

That a small increase in $A \mathrm{~L}$ leads to perception of interruption in the center noise would not be predicted if continuity was produced by relations between total neural effects of $B$ and $A$ stimuli, because total neural effect due to $B$ should still be larger than that due to $A$ even after the increase in $A \mathrm{~L}$ intensity.

\section{EXPERIMENT 3}

This experiment was performed to obtain a comparison between monaural and binaural continuity effects at two different stimulus durations.

\section{Method}

In this experiment, for the binaural stimulus, the $A \mathrm{~L}$ noise stimulus was omitted, giving the stimulus pattern shown in Figure 3a. $B$ and $A$ stimuli were presented in alternation.

$B \mathrm{~L}$ and $A \mathrm{R}$ were noise stimuli set at $60 \mathrm{~dB}(\mathrm{~A}) . B \mathrm{R}$ intensity was adjusted from an intensity that gave continuity for $A \mathrm{R}$ (perceived localized to the right) to a lower intensity that just produced intermittence. For comparison, a monaural stimulus, composed of $B R$ and $A \mathrm{R}$, was presented to the right ear (Figure $3 \mathrm{~b}$ ). $B \mathrm{R}$ was decreased in intensity until the subject perceived intermittence.

Four determinations of intermittence were made for each condition, using the method of limits. Before each threshold determination, the intermittence rate the subject was supposed to listen for was demonstrated. Threshold determinations were made for $B$ (and

Table 2

Average Decibel Changes to Produce Effects on A Sound of Figure 2

\begin{tabular}{cccccc} 
& \multicolumn{2}{c}{ Interruption Perceived } & & \multicolumn{2}{c}{ Continuous Sound Lost } \\
\cline { 2 - 3 } \cline { 5 - 6 } Experiment & $A \mathrm{~L}$ Increase & $B \mathrm{~L}$ Decrease & & $A \mathrm{~L}$ Decrease & $\boldsymbol{B}$ L Increase \\
\hline 2 & 2.8 & 3.2 & & 7.2 & 7.0 \\
\hline
\end{tabular}

$B \quad \mid \ln$

$\ln$

$\mathbf{n} \mid$

A $\quad$ n

$\mid R$

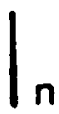

$3 a$

$3 b$

$3 c$

Figure 3. Schematic diagrams of initial stimulus conditions. B and A represent stimuli alternating in time, delivered to left and right earphones. The right-hand line of a pair represents a stimulus delivered to the right earphone; the left-hand line of a pair represents a stimulus delivered to the left earphone. $n=$ noise.

A) stimulus durations of 45 and $245 \mathrm{msec}$, in an order counterbalanced across subjects.

In addition, each subject was checked with $B \mathrm{~L}$ and $A \mathrm{R}$ alternating (Figure 3c) and asked whether the stimulus on the right was perceived as continuous or intermittent. The subjects always perceived the right-localized stimulus as intermittent, at both the 45 and 245 -msec stimulus durations.

Ten undergraduate subjects took part in this experiment. In this and subsequent experiments, the subjects had to have had some form of musical training, vocal or instrumental.

\section{Results}

Table 3 gives the results of Experiment 3. Values given for the 10 subjects are average $\mathrm{dB}(\mathrm{A})$ values at which intermittence was perceived. At the $45-\mathrm{msec}$ duration, monaural and binaural thresholds are not significantly different: the intensity relations between $B \mathrm{R}$ and $A \mathrm{R}$ required for producing intermittence are not significantly affected by the presence of $B \mathrm{~L}$. At the 245 -msec duration, the monaural and binaural thresholds are significantly different (signed ranks test, $p<.01$ ). It appears that at $245 \mathrm{msec}$, the presence of $B \mathrm{~L}$ has reduced the effective intensity of $B R$ in producing continuity in $A R$.

At both 45 and $245 \mathrm{msec}$, in the binaural pattern of Figure $3 \mathrm{a}$, continuity for $A \mathrm{R}$ is controlled by $B \mathrm{R}$, rather than by total $B \mathrm{~L}$ and $B \mathrm{R}$ stimulus effects, since intermittence is produced (and continuity lost) when total $B$ stimulus input effects are still larger than total $A$ stimulus input effects.

\section{EXPERIMENT 4}

From results with the noise patterns used in the first two experiments, it was inferred that continuity in the $A$ binaural stimulus occurred only when intensity relations in each ear were such as to produce continuity for the $A$ component in each ear separately. Experiment 4 was designed to check this inference further.

\section{Method}

Twelve subjects were tested with patterns schematically depicted in Figure 4. $B$ stimuli were $245 \mathrm{msec}$ tones of $500 \mathrm{~Hz}$, set at a level of $56 \mathrm{~dB}(\mathrm{~A})$. The $A$ stimulus was a tone of $550 \mathrm{~Hz}$ at a duration of $245 \mathrm{msec} . B$ and $A$ stimuli were presented in alternation. 
Table 3

Average BR Decibel Values to Produce

Intermittence in A Sound of Figure 3

\begin{tabular}{cccccc}
\hline \multicolumn{2}{c}{$45 \mathrm{msec}$} & & \multicolumn{2}{c}{$245 \mathrm{msec}$} \\
\cline { 2 - 3 } \cline { 5 - 6 } 1 ear & 2 ears & & 1 ear & 2 ears \\
\hline 58.1 & 58.2 & & 58.4 & 62.4 \\
\hline
\end{tabular}

Half of the subjects started with Pattern 4b. The intensity of $A \mathrm{R}$ was decreased until the subject judged that the higher pitched stimulus $(A)$ sounded continuous. This was done four times. The level of $A \mathrm{R}$ was then set $2 \mathrm{~dB}$ softer than the average continuity threshold, and $A \mathrm{~L}$ was introduced (Pattern 4a). Threshold for continuity for the binaural $A$ tone was then determined by changing the intensity of $A$ L. Finally, threshold for continuity was determined for $A$ when only $A L$ was present (Pattern $4 c$ ). We were interested in comparing these last two thresholds: was binaural $A$ perceived as continuous only when $B / A$ intensity relations were sufficient to produce continuity in the $A$ component in each ear?

The other half of the subjects followed a similar procedure, but were tested with Pattern $4 c$, then with Pattern $4 a$, and finally with Pattern 4b.

\section{Results}

The average difference between the threshold for continuity of an $A$ component when tested in Pattern $4 \mathrm{~b}$ or Pattern $4 \mathrm{c}$ and when tested in Pattern $4 \mathrm{a}$ was $.1 \mathrm{~dB}$. A statistical signed ranks test indicated that the differences obtained were not significant. These results are in agreement with our previous conclusion that continuity for a binaural $A$ stimulus is perceived only when a continuity condition exists for each $\boldsymbol{A}$ component separately. It should be noted that this conclusion has been obtained when the frequency of $A \mathrm{~L}$ equals the frequency of $A \mathrm{R}$, and does not hold when these frequencies are very different.

\section{EXPERIMENT 5}

In Experiment 4 , Pattern $4 \mathrm{~b}$, when $A \mathrm{R}$ was sufficiently lower in intensity than $B \mathrm{R}, A \mathrm{R}$ was heard as continuous. $A \mathrm{R}$ did not pick up the $B \mathrm{R}$ pitch. In Experiment 5, it will be shown that when speech is used, $A \mathrm{R}$ does pick up the $B R$ speech.

\section{Method}

In Experiment 5 speech was tested with patterns similar to those used in Experiment 3 with noise (see Figures $5 a$ and 5b). $B$ and
B $500+1 \mid$
$+1$

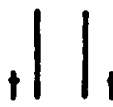
A 550

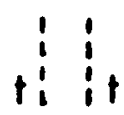
40

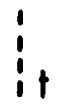
$4 \mathrm{~b}$

Figure 4. Schematic diagrams of initial stimulus conditions. B and A represent stimuli alternating in time. The right-hand line of a pair represents a stimulus delivered to the right earphone; the left-hand line of a pair represents a stimulus delivered to the left earphone. $t=$ tone. Numerals 500 and 550 refer to frequencies of tone stimuli. A dashed line represents a lower intensity than a solid line.
$A$ stimuli were alternated, and the duration for $B$ and $A$ stimuli was $245 \mathrm{msec}$. Onset and offset time for the stimuli was $5 \mathrm{msec}$. The peak level of the $B L$ and $A R$ speech was near $64 \mathrm{~dB}(A)$. Speech was recorded on a Magnacorder tape recorder (Model 728), using a VU meter to maintain constancy of peak levels. The speech was prose, read form James Thurber's (1973) The Beast in Me.

Twelve undergraduate subjects were tested with the pattern of Figure 5a. The intensity of $B R$ was increased in 5-dB steps until the subject reported that the speech heard to the right was perceived as continuous. This measure was performed twice, and the decibel setting at which continuity was reported was recorded.

Ten additional subjects were tested with Patterns $5 a$ and 5 b. Levels of $B \mathrm{~L}$ and $A \mathrm{R}$ were set as previously, but $B \mathrm{R}$ in Pattern 5a was set $10 \mathrm{~dB}$ higher. The subjects were asked to listen carefully to the words of the speech heard to the right, and to report whether they heard any pieces missing in the words. Each condition was tested twice.

\section{Results}

The first 12 subjects heard the speech at the right as continuous for the binaural stimulus (Figure 5a) when $B R$ intensity was close to the intensity of $A \mathrm{R}$, or higher than that of $A \mathrm{R}$. The threshold for continuity was reached when $B R$ intensity was on the average $1.4 \mathrm{~dB}$ greater than that of $A \mathrm{R}$.

In the additional group of 10 subjects tested with Patterns $5 a$ and $5 b, 9$ of the 10 consistently reported hearing pieces of words missing in the words heard at the right for Pattern $5 b$ but no pieces missing for the binaural pattern of Pattern 5a, where $B R$ was present and set at an intensity $10 \mathrm{~dB}$ higher than that of $A \mathrm{R}$ (and $B \mathrm{~L}$ ). In the latter case, it is significant that the $B$ speech pattern (but not loudness level) was being picked up to complete the $A \mathrm{R}$ speech perceived at the right.

\section{DISCUSSION OF EXPERIMENTS 1-5}

To explain these continuity effects, we will use modifications of theoretical approaches earlier proposed by others. We will start with a brief review of these earlier explanations of monaural continuity effects.

For tonal continuity, Houtgast (1972) postulated that the tone was perceived as continuous when the transition from masker to tone caused no increase in nervous activity in any frequency region (occupied by the neural representation of the tone). Verschurre (1978) used a similar hypothesis-that continuity is perceived if no information indicating that a signal is interrupted is available to any channel of the auditory system. He further postulated that the gap appearing in the signal was filled with the most probable signal. These hypotheses are similar to those proposed by Warren, Obusek, and Ackroff (1972): "If there is contextual evidence that a sound may be present at a given time, and if the peripheral units stimulated by a louder sound include those which would be stimulated by the anticipated fainter sound, then the fainter sound may be heard as present"' (p. 1151).

Although it may be clear in the case of interrupted speech sounds what speech sound would be anticipated (or would be most probable) in the gaps filled by noise, a most probable signal cannot be clearly defined to ex- 


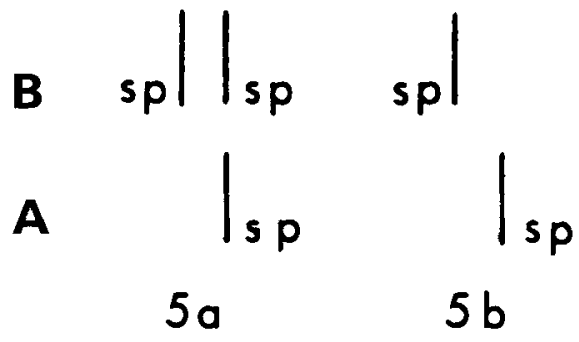

Figure 5. Schematic diagrams of initial stimulus conditions. B and A represent stimuli alternating in time. The right-hand line of a pair represents a stimulus delivered to the right earphone; the left-hand line of a pair represents a stimulus delivered to the left earphone. sp $=$ speech.

plain simple tonal continuity. There, the $B$ stimulus in the gaps is even more probable than the $A$ stimulus-it is actually physically present! In addition, simple conscious expectancies may not predict the result of the synthesis of information. Thurlow and Erchul (1978) noted that interrupted FM sweeps can sound like single notes (do-remi) when proper sweep rate and duration are used. However, when noise fills the gaps, the perception changes to a continuous pitch-sweep upward. The synthesized perception does not involve filling in continuations of do-re-mi, nor can the final perception be considered a filling-in of what is "expected."

The framework within which we will interpret the present results is similar to that of theories described above. It involves the general hypothesis that when no gap can be detected in a stimulus (related to a decrease in effective intensity of that stimulus) there is a cortical "instruction" to synthesize available information about that stimulus over a longer time period (than when a gap is detected). (1) If $A$ pieces occur with no evidence of gaps but with strong serial and/or contextual cues as to what might appear in the $B$-filled gaps, then we get what Warren (1970) has described as "phonemic substitution." Verschurre and Brocaar (1983) state "with intervening noise, some speech recognition process necessarily fills in the gaps in the signal, the redundancy level determining the possibilities offered to the auditory system in this filling-in process"' (p. 239) (2) If $A$ pieces occur with no evidence of gaps and with no serial and/or contextual cues as to what might appear in the $B$-filled gaps, then the brain synthesizes a continuous perception from what information is available. This implies that pattern, loudness, and localization information presented in the $A$ pieces are sufficient for generating a complete perception; and it is not necessary to synthesize additional pieces to fill the gaps, a process that would be particularly difficult when complex $A$ stimuli are involved, such as pulses in a temporal pattern (Thurlow \& Erchul, 1978).

Additions to these descriptive hypotheses are needed for an explanation of binaural continuity effects. (1) Noise experiments and tone experiments: It is assumed that the final continuous perception that is synthesized has the location "tag"'of the $A$ stimulus and a loudness "tag" related to the combination of $A \mathrm{~L}$ and $A \mathrm{R}$ binaural components. How is the decision on continuity made? It is assumed that information on presence (or nonpresence) of a "gap" is provided from each ear to a central processor. When $A \mathrm{~L}$ and $A \mathrm{R}$ frequencies are the same (as in our experiments with noise and tone), evidence from both ears must indicate continuity for $A$ to be perceived as continuous. This result is not due to a simple additive combination of components (from each ear ) each of which has been made continuous. In the noise pattern of Figure $3 a$, for example, when $B R$ is made sufficiently less intense than $A \mathrm{R}$ to make $A \mathrm{R}$ sound interrupted, a continuous $B R$ component in the median-plane location is not heard, although it can be clearly heard at the right if we listen to right-ear stimuli alone. This result is assumed to occur because the $B \mathrm{~L}$ component at the median-plane location is sending a "gap" signal to the central processor. (2) Speech stimuli: It is assumed that when the subject listens to the $A$ stimulus of Experiment 5 (see Figure 5a), a continuity decision is reached for $A \mathrm{R}$ when no decrease in $A R$ can be detected (cued by the relative effective intensities of $B \mathrm{R}$ and $A \mathrm{R})$. $A \mathrm{R}$ then automatically picks up the available $B$ pattern (presumably aided by associative linkages between $B \mathrm{R}$ and $A \mathrm{R}$ ). If relative intensity cues do not indicate continuity, each $A$ piece tends to be perceived separately.

\section{ADDITIONAL TYPES OF BINAURAL CONTINUITY EFFECTS}

\section{$A$ "Center" Noise Perceived with Alternating $A R$ and $B L$ Stimuli}

Thurlow and Marten (1962) observed that when two thermal noise pulses were presented alternately in time on either side of the median plane, a number of subjects heard an additional softer continuous noise located in the median plane, provided that the rate of alternation was sufficiently high. This perception of noise in the median plane can also be produced, when earphones are used, by broadband noise stimuli that alternate in left and right locations-locations produced by small intensity differences at the two ears or by time-of-arrival differences at the two ears. We found that combining a time lead with an intensity difference $(800-\mathrm{msec}$ lead with a 5 -dB higher intensity for $B \mathrm{R}$ versus $B \mathrm{~L}$ and for $A \mathrm{~L}$ versus $A \mathrm{R}$, for example) removed the center noise. These observations were made with the subjects of Experiment 1, in which pulse durations were near $40 \mathrm{msec}$. Erchul (1979) found that increasing intensity differences alone $(B \mathrm{R}>B \mathrm{~L}$, and $A \mathrm{~L}$ $>A \mathrm{R}$ ) to $14.8 \mathrm{~dB}$, on the average, caused the center noise to disappear. The center noise is clearly a different kind of continuity effect. It occurs at a location that is different from the subjective location of either of the stimulus pulses used to produce it. It may arise in a neural localization mechanism. A time lead alone, for each of the alternating stimuli, may allow some neural units that are sensitive to intensity differences at the two ears to signal 
<smiles>S=[SbH]</smiles>

A

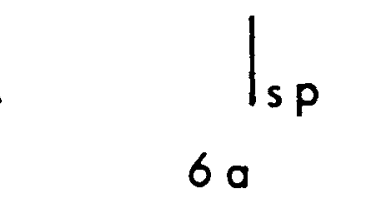

$$
\text { sp } \mid
$$

$\mid \begin{aligned} & R \\ & n\end{aligned}$<smiles>O=S(=O)(O)O</smiles>

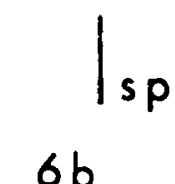

$\mid \begin{aligned} & R \\ & s p\end{aligned}$

$6 c$
$6 d$

Figure 6. B and A represent stimuli alternating in time. The right-hand line of a pair represents a stimulus delivered to the right earphone; the left-hand line of a pair represents a stimulus delivered to the left earphone. Other abbreviations: $n=$ noise; $\mathbf{s p}=$ speech.

a median-plane location; a small intensity difference alone, for each of the alternating stimuli, may allow some neural units that are primarily sensitive to time differences at the two ears to signal a median-plane location.

\section{Noise Facilitation of Other-Ear Pattern Pickup}

Another type of continuity effect occurs with the stimulus pattern shown in Figure 6a. The noise stimulus $B R$ facilitates pickup of the $B \mathrm{~L}$ speech in the opposite ear by $A R$. This effect of noise has not been reported previously, and it will be described in some detail below.

\section{EXPERIMENT 6}

\section{Method}

Sixteen undergraduate subjects were tested with the patterns depicted in Figure 6. $A \mathrm{R}$ and $B \mathrm{~L}$ speech was at a level such that the peaks were close to $64 \mathrm{~dB}(\mathrm{~A})$. The tape-recorded speech was the same as that used in Experiment 5 . The thermal noise stimulus $(B R)$ was set at a level of $78 \mathrm{~dB}(\mathrm{~A}) . B$ and $A$ stimuli were alternated in time, and each stimulus had a duration of $345 \mathrm{msec}$. Onset and offset times for the stimuli were $5 \mathrm{msec}$.

The subjects were asked to listen to the words heard to the right (to ignore speech heard in any other location), and to report whether the words had any pieces missing. Patterns $6 a, 6 b$, and $6 c$ were used five times in sets of three. (The subjects were additionally tested with Pattern 6d, and asked whether they heard any speech sounds on the right; none did.)

\section{Results}

The number of "yes" responses was tabulated for each condition, for each subject. A "yes" response indicated that the subject heard some pieces missing in the words heard in the right location. Median number of "yes" responses (out of 5 possible) was 1 for Pattern 6a, 5 for Pattern 6b, and 4.5 for Pattern $6 \mathrm{c}$. Fifteen of the 16 subjects gave fewer "yes" responses for Pattern 6a than for Pattern 6b. A signed ranks test showed this result to be significant $(p<.01)$. Thus, BL speech is not picked up by $A \mathrm{R}$ as well when $B \mathrm{R}$ noise is absent. This is not a monaural effect due to subjects' filling-in of speech sounds when $B \mathrm{R}$ noise is added to $A \mathrm{R}$ speech: 12 of the 16 subjects gave fewer "yes" responses for Pattern 6a than for Pattern 6c. A signed ranks test showed this result to be significant $(p<.01)$.

In an earlier, exploratory experiment run with 13 subjects, 11 subjects had given fewer "yes" responses with
Pattern 6a than with Pattern $6 \mathrm{~b}$, and 12 had given fewer "yes" responses with Pattern 6a than with Pattern 6c.

\section{EXPERIMENT 7}

This experiment demonstrated that pickup of a $B \mathrm{~L}$ tone by $A \mathrm{R}$ can be facilitated by $B \mathrm{R}$ noise.

\section{Method}

Fifteen subjects were tested with the patterns shown in Figures $7 \mathrm{a}$ and $7 \mathrm{~b} . B$ and $A$ stimuli alternated in time, and each had a duration of $245 \mathrm{msec}$. $B \mathrm{~L}$ had a frequency of $550 \mathrm{~Hz}$ and $A \mathrm{R}$ had a frequency of $500 \mathrm{~Hz}$. Each was at a level of $57 \mathrm{~dB}(\mathrm{~A})$. BR was a 78$\mathrm{dB}(\mathrm{A})$ thermal noise stimulus. Onset and offset times for the stimuli were $5 \mathrm{msec}$.

For each condition, the subjects were asked to listen to what they heard on the right and to ignore anything they heard in other locations. They were asked to report whether they heard two alternating pitches in the right ear or only one pitch. A demonstration of two alternating pitches versus one pitch was given initially by presenting stimuli to one ear alone.

The two stimulus conditions were each presented seven times, in sets of two (7a and 7b). (Subjects were finally checked with Pattern $7 \mathrm{c}$, to find whether they heard any tone on the right; they did not.)

\section{Results}

Responses of "two alternating pitches" were tabulated for each subject. A median of 3.5 of such responses (out of 7) was found for the condition in which noise was present (Pattern 7a); a median of 0 was obtained when noise was not present (Pattern $7 \mathrm{~b}$ ). Thirteen of the 15 subjects gave more responses of "two alternating pitches"

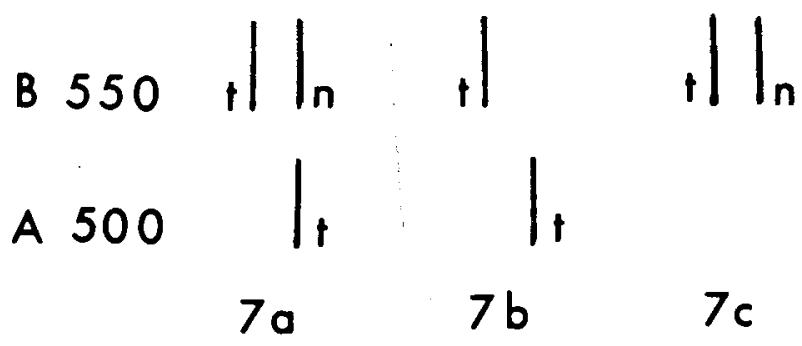

Figure 7. B and A represent stimuli alternating in time. The righthand line of a pair represents a stimulus delivered to the right earphone; the left-hand line of a pair represents a stimulus delivered to the left earphone. Other abbreviations: $n=$ noise; $t=$ tone. Numerals $\mathbf{5 0 0}$ and $\mathbf{5 5 0}$ refer to frequencies of tone stimuli. 
when $B \mathrm{R}$ noise was present than when it was not. This result was significant by signed ranks test $(p<.01)$. Although this effect was strong in some subjects, it was weak or nonexistent in others. There is no strong associative connection here between $A \mathrm{R}$ and $B \mathrm{~L}$ that would increase the $B \mathrm{~L}$ pattern pickup.

In earlier exploratory experiments, there had been some $B \mathrm{~L}$ tone pickup effects when $A \mathrm{R}$ was $500 \mathrm{~Hz}$ and $B \mathrm{~L}$ was 550,600 , or $1000 \mathrm{~Hz}$ and also when $A \mathrm{R}$ was $1000 \mathrm{~Hz}$ and $B L$ was 1100,1200 , or $2000 \mathrm{~Hz}$. However, only a minority of subjects showed these $B \mathrm{~L}$ tone pickup effects.

\section{DISCUSSION OF EXPERIMENTS 6 AND 7}

A stimulus in one ear can pull the localization of another stimulus in the opposite ear toward a more central location, provided that the two stimuli are not too different in frequency (Thurlow \& Elfner, 1959b). Noise in one ear can have a similar effect on a stimulus in the other ear (Butler \& Naunton, 1962, 1964; Egan, 1948; Warren \& Bashford, 1976).

The results of Experiment 6 indicate that there can be facilitation of pickup of a speech component $(B \mathrm{~L})$ from the opposite ear by $A R$ when noise is used for $B R$ (see Figure 6a). It might be hypothesized that $B R$ noise produces a "localization link" between $B \mathrm{~L}$ and $A \mathrm{R}$. Frequency components in the noise (of varying amplitude) could pull over corresponding frequency components in the speech toward the right, producing a wide distribution of localization positions for the speech frequency components from left to right. Even though the $B \mathrm{~L}$ stimulus, when tested with $B R$ noise alone, is localized to the center or left of center (presumably on the basis of the median of the distribution of localization effects), there might be a localization link from left to right caused by the whole distribution of localization effects that enables the $B \mathrm{~L}$ speech pattern to be picked up by $A \mathrm{R}$.

The $B \mathrm{~L}$ pattern pickup reminds us of the results from numerous studies of “duplex perception"' (first reported by Rand, 1974), where a pattern component is picked up by the opposite ear but can be heard in the original ear also. We checked with 10 additional subjects, using the stimuli of Experiment 7 (Figure 7a), and found that the $B L$ tone could be heard in a center or a left location at the same time that it was being picked up by the right-ear $A \mathrm{R}$ stimulus to form an alternating pitch pattern. It is possible that a "localization linkage" plays a role in some experiments on duplex perception, with a tone in one ear being picked up by a chord in the other ear (see Collins, 1985; Pastore, Schmuckler, Rosenblum, \& Szczesiul, 1983). Collins (1985) found only a low incidence of duplex perception.

Further checks were conducted with these 10 subjects to see whether the $B \mathrm{~L}$ pattern pickup (in the situation of Figure 7a) could occur when $A \mathrm{R}$ was perceived as continuous. Using right-ear stimuli alone and Pattern $7 \mathrm{a}, A \mathrm{R}$ intensity was reduced to produce perception of continuity. Then the subject listened to Pattern $7 \mathrm{a}$ with $B \mathrm{~L}$ present in the left ear. Pattern pickup could be observed in this condition. An "instruction" to synthesize available information, when no gap cue is present, may allow utilization of information available from localization linkage.

\section{REFERENCES}

Butler, R. A., \& Naunton, R. F. (1962). Some effects of unilateral masking upon the localization of sound in space. Joumal of the Acoustical Society of America, 34, 1100-1107.

Butler, R. A., Naunton, R. F. (1964). Role of stimulus frequency and duration in the phenomenon of localization shifts. Journal of the Acoustical Society of America, 36, 917-922.

Collins, S. C. (1985). Duplex perception with musical stimuli: A further investigation. Perception \& Psychophysics, 38, 172-177.

EGAN, J. P. (1948). The effect of noise in one ear upon the loudness of speech in the other. Journal of the Acoustical Society of America, $20,58-62$.

ElFNER, L. F., \& CASkeY, W. E. (1965). Continuity effects with alternately sounded noise and tone signals as a function of manner of presentation. Joumal of the Acoustical Society of America, 38, 543-547.

ELFNER, L. F., \& HoMICK, J. L. (1966). Some factors affecting the continuity in alternately sounded tone and noise signals. Journal of the Acoustical Society of America, 40, 27-31.

ElFNer, L. F., \& Homick, J. L. (1967). Continuity effects with alternately sounding tones under dichotic presentation. Perception \& Psychophysics, 2, 34-36.

ERCHUL, W. P. (1979). Two types of auditory continuity effects: A study using skilled listeners. Unpublished senior honors thesis, Psychology Department, University of Wisconsin, Madison.

Houtgast, T. (1972). Psychophysical evidence for lateral inhibition in hearing. Joumal of the Acoustical Society of America, 51, 1885-1894.

Pastore, R. E., Schmuckler, M. A., Rosenblum, L., \& Szczesiul, R. (1983). Duplex perception with musical stimuli. Perception \& Psychophysics, 33, 469-474.

RAND, T. C. (1974). Dichotic release from masking for speech. Journal of the Acoustical Society of America, 55, 678-680.

ThURBER, J. (1973). The beast in me. New York: Harcourt Brace Jovanovich.

Thurlow, W. R., \& Elfner, L. F. (1959a). Continuity effects with alternating tones. Journal of the Acoustical Society of America, 31, 1337-1339.

Thurlow, W. R., \& ElfNer, L. F. (1959b). Pure-tone cross-ear localization effects. Journal of the Acoustical Society of America, 31, 1606-1608.

ThuRLOW, W. R., \& ERChUL, W. P. (1978). Understanding continuity effects with complex stimuli. Journal of the American Auditory Society, 4, 113-115.

Thurlow, W. R., \& Marten, A. E. (1962). Perception of steady and intermittent sounds with alternating noise-burst stimuli. Journal of the Acoustical Society of America, 34, 1853-1858.

VerschurRe, J. (1978). Auditory excitation patterns. Delft: W. D. Meinema B.V.

VerschURRE, J., \& BROCAAR, M. P. (1983). Intelligibility of interrupted meaningful and nonsense speech with and without intervening noise. Perception \& Psychophysics, 33, 232-240.

WARREN, R. M. (1970). Perceptual restoration of missing speech sounds. Science, 167, 392-393.

WARrEN, R. M. (1982). Auditory perception. New York: Pergamon.

WARREN, R. M., Bashford, J. A. (1976). Auditory contralateral induction: An early stage in binaural processing. Perception \& Psychophysics, 20, 380-386.

WARren, R. M., ObuSEk, C. J., \& ACKroff, J. M. (1972). Auditory induction: Perceptual synthesis of absent sounds. Science, 176, 1149-1151.

(Manuscript received February 25, 1986; revision accepted for publication February 14, 1987.) 